\title{
Calidad de vida relacionada con la salud oral en una población venezolana en el Perú
}

\section{Quality of life related to oral health in a Venezuelan population in Peru}

M. R. Valenzuela Ramos*, R. D. Scipión Castro**, J. P. Portocarrero Mondragón***

\section{RESUMEN}

OBJETIVO. El objetivo de la presente investigación está enfocado en evaluar la calidad de vida relacionada con la salud oral en una población venezolana en el Perú.

MATERIAL Y METODOS. Se trata de un estudio analítico transversal realizado entre los meses de setiembre a noviembre del 2019 a la población de nacionalidad venezolana. Participaron un total de 880 nacionales venezolanos quienes participaron voluntariamente del estudio, de los cuales $45.9 \%$ (379) eran de sexo femenino y $54.1 \%$ (501) de sexo masculino. Se realizó un examen clínico a fin de determinar las variables clínicas: el índice CPOD, el índice de higiene oral y el índice gingival. Para evaluar la calidad de vida relacionada con la salud oral se utilizó el Perfil de Impacto en la Salud Oral OHIP-14 que consta de 49 preguntas agrupadas en 7 dimensiones que son la limitación funcional, dolor físico, disconfort psicológico, incapacidad física, incapacidad psicológica, incapacidad física y en desventaja. Para si se da un puntaje de 1 y no 0 . Así, a un mayor puntaje se obtiene la calidad de vida es peor.

RESULTADOS. La relación de la calidad de vida con salud se verifica que un total del $27 \%$ (116.869) fueron respuestas positivas y un $72 \%(31,434)$ respuestas negativas.

CONCLUSIONES. En relación a la calidad de vida relacionada con la salud oral se obtuvo que la población estudiada tiene buena calidad de vida, representada por más de la mitad de los encuestados.

PALABRAS CLAVE: Calidad, Vida, Salud, Oral.

ABSTRACT
AIM. Theobjective of thepresentinvestigationisfocusedonevaluatingthequality of liferelated to oral health in
a Venezuelanpopulation in Peru.
MATERIAL AND METHODS. Thisis a cross-sectionalanalyticalstudycarriedoutbetweenthemonths of Sep-
tember and November of 2019 to thepopulation of Venezuelannationality. A total of 880 Venezuelannatio-
nalsparticipatedwhovoluntarilyparticipated in thestudy, of which $45.9 \%$ (379) werefemale and $54.1 \%(501)$
male. A clinicalexaminationwasperformed to determine theclinical variables: the CPOD index, the oral hy-
gieneindex and the gingival index. To assessthequality of liferelated to oral health, checkthe OHIP-14 Oral
HealthImpactProfile, whichconsists of 49 questionsgroupedinto 7 dimensionsthat are functionallimitation,
physicalpain, psychologicaldistress, physicaldisability, psychologicaldisability, physical and disadvanta-
geddisabilityForitself a score of 1 isgiven and not 0 . Thus, a higher score gets the quality of life is worse.
RESULTS. The relationship of quality of life with health verifies that a total of $27 \%(116,869)$ were positive
responses and $72 \%$ (31,434) negative responses.
CONCLUSIONS. Regarding the quality of life related to oral health, it was obtained that the population
studied has a good quality of life, represented by more than half of the respondents.
* Directora de Escuela Estomatología - Universidad Señor de Sipán.
** Docente Universidad Señor de Sipán.


KEY WORDS: Quality, Life, Health, Oral.

Fecha de recepción: 7 de enero de 2020

Fecha de aceptación: 10 de febrero de 2020

M. R. Valenzuela Ramos, R. D. Scipión Castro, J. P. Portocarrero Mondragón. Calidad de vida relacionada con la salud oral en una población venezolana en el Perú. 2020; 36, (4): 186-190.

\section{INTRODUCCIÓN}

Según Organización de las Naciones Unidas (ONU) el número de migrantes internacionales en el mundo ascendió en los últimos 10 años a 272 millones. De dicha cifra 4,769,498aproximadamente pertenece a la población venezolana. Se estima que568,973 venezolanos viven en el Perú. ${ }^{1,23}$

La recesión económica sumada a las altas tasas de desempleo en Venezuelaha provocado grandes flujos migratorios, principalmente a países de la región, donde se desenvuelven trabajando en sectores poco calificados. ${ }^{4}$

Dado que la inmigración venezolana es un fenómeno nuevo se conoce poco o nada sobre la calidad de vida relacionada con la salud oral de dicha población en el Perú. Los asuntos relacionados a las inmigraciones como es la salud son desafíos y temas preocupantes que enfrentan los gobiernos en la actualidad.

Esta población se ve forzada a la adaptación de hábitos, costumbres y mitos culturales que intervienen en la percepción de salud del país que los acoge. A nivel mundial existe una inequidad en salud, resultado de la desigualdad en la distribución de los recursos sociales y económicos. Esta inequidad se ve reflejada en la salud bucal, autores como Mouradian ${ }^{5}$ manifiestan que para eliminar estas desigualdades en relación a la salud bucal es necesario integrar esta dentro de la salud general. ${ }^{6}$

Ha sido demostrado que una condición socioeconómica desfavorable influye en la aparición de enfermedades orales. Sin embargo, ello no es suficiente para explicar un impacto en el ámbito multidimensional y multifactorial de la salud oral en la calidad de vida, que es conocida como la calidad de vida relacionada a la salud oral. ${ }^{7}$

El perfil de impacto de la salud oral (OHIP-14) es uno de los instrumentos más utilizados para medir la calidad de vida relacionada con la salud oral. Este instrumento fue desarrollado con el fin de examinar el impacto de los problemas orales en la vida de una persona, en especial en personas adultas. ${ }^{8}$

En la actualidad, la inmigración de la población venezolana es alta, siendo Perú el segundo país de acogida preferido de los inmigrantes venezolanos. En este sentido, el objetivo de la presente investigación está enfocada en evaluar la calidad de vida relacionada con la salud oral en una población venezolana en el Perú.

\section{MATERIAL Y MÉTODOS}

Se trata de un estudio analítico transversal realizado entre los meses de setiembre a noviembre del 2019 a la población de nacionalidad venezolana que acudían al centro de prácticas pre-profesionales de la Universidad Señor de Sipán. Participaron un total de 880 nacionales venezolanos quienes participaron voluntariamente del estudio, de los cuales $45.9 \%(379)$ eran de sexo femenino y $54.1 \%(501)$ de sexo masculino. Se realizó un examen clínico a fin de determinar las variables clínicas: el índice CPOD, el índice de higiene oral $\mathrm{y}$ el índice gingival.

El examen de caries dentalfue realizado por especialistas en un sillón dental y con luz blanca, después de ser realizado el cepillado dental. La caries dental se diagnosticó usando el protocolo de la Organización Mundial de la Salud utilizando en índice CPO-D (dientes cariados, perdidos debido a la caries dental y dientes obturados). Ello se utilizó la cuantificación de la OMS para en índica CPOD, donde muy bajo se califica de 0.0 a 1.1 , bajo de 1.2 a 2.6, moderado de 2.7 a 4.4 y alto de 4.5 a $6.5 .9,10$

Se utilizó el índice gingival de Loe y Silnes para evaluar la presencia y agudeza de la inflamación gingival donde se puntua de 0 a 3 donde 0 indica la ausencia de placa y se transcribe como normal, 1 cuando no hay placa a la vista, pero si existe placa cuando se realiza el pasaje de sonda 
por el área dentogingival lo que llega a ser leve, 2 hay placa bacteriana simple vista puntuándolo como moderado y 3 placa bacteriana a la vista rodeando el diente, incluso por los espacios interdentales, se puede o no apreciar cálculos llegando a ser intensa. ${ }^{11,12}$

El índice de higiene oral se utilizó con el fin de evaluar los hábitos de higiene oral del paciente. Mide la superficie del diente cubierta con desechos y cálculos, los componentes de este índice son depósitos blandos y duros. Se refiere a blando a placa bacteriana y restos de alimentos y pigmentos, y depósitos duros a los cálculos dentales. Son medidos primeras molares e incisivos centrales. Los ítems para evaluar criterios de depósitos duros y blandos van del 0 al 3. Así, para los criterios blandos 0 no hay depósitos ni pigmentaciones, 1 existen depósitos en no más del $1 / 3$, o hay pigmentación, 2 existen depósitos en no más del $1 / 3$ pero menos de $2 / 3$ y 3 cuando los depósitos cubren más de $2 / 3$ de la superficie.Para evaluar los criterios de los depósitos duros se considera 0 cuando no hay tártaro, 1 cuando el tártaro supragingivalno cubre más de $1 / 3$ de la superficie dentaria, 2 cuando el tártaro supragingival cubre más de $1 / 3$ de la superficie dentaria y menos de $2 / 3$ de esta superficie y tres cuando cubre más de 2/3 de la superficie dentaria. Para realizar el cálculo de los índices se suma los códigos y se divide entre el número de dientes. La cuantificación que se obtiene de ellos es; 0.0 - 1.0 optimo, 1.1 a 2.0 regular, 2.1 a 3.1 malo y 3.1 a mas muy malo. ${ }^{13}$

Para evaluar la calidad de vida relacionada con la salud oral se utilizó el Perfil de Impacto en la Salud Oral OHIP-14 que consta de 49 preguntas agrupadas en 7 dimensiones que son la limitación funcional, dolor físico, disconfort psicológico, incapacidad física, incapacidad psicológica, incapacidad física y en desventaja. Para si se da un puntaje de 1 y no 0 . Así, a un mayor puntaje se obtiene la calidad de vida es peor. ${ }^{8}$

Luego se evaluó cada una de las dimensiones del instrumento OHIP-14 la calidad de vida relacionada con la salud oral en relación al índice CPOD, al índice de higiene oral y al índice gingival.

\section{RESULTADOS}

Los resultados obtenidos en el presente estudio muestran que en $29.5 \%$ (260) de la población estudiada tiene un índice CPO-D muy bajo, el 45\%(396) bajo, 22.3\% (196) moderado y un 3.2\% (28) alto.

En relación al índice gingival se puede verificar que el 32,3\% (284) se cuantifica dentro de una categoría normal, el 57.5\% (506) leve, 9.8\% (86) moderado y $0.5 \%(4)$ intenso.

El índice de higiene oral se verifica óptimo para el $16.6 \%$ (146), regular 22\% (194), 18.6\% (164) malo y muy malo $42.7 \%$ (376).

La relación de la calidad de vida con salud se verifica que un total del 27\% (116.869) fueron respuestas positivas y un $72 \%(31,434)$ respuestas negativas.

En la tabla 01 se observa que existe relación entre la dimensión limitación funcional y el CPOD bajo ( $p$ - valor 0.033), el índice gingival normal $(p-$ valor 0.004 ), y el índice de higiene oral muy malo ( $p$ - valor 0.022).

En relación a la dimensión limitación funcional y CPOD bajo un $20.3 \%$ responde afirmativamente a la pregunta ¿Has tenido problemas pronunciando alguna palabra por problemas con tus dientes, boca o prótesis?

En la dimensión limitación funcional y el índice gingival normal el $20.7 \%$ responde afirmativamente a la pregunta chas tenido dificultades mordiendo algún alimento por problemas con tus dientes, boca o prótesis?

Cuando se pregunta sobre la limitación funcional y el índice de higiene oral un $22.1 \%$ responde positivamente a la pregunta ¿Has notado un diente que no se ve bien?

TABLA 01. Relación entre la limitación

funcional y los indicadores de salud bucal

Variables correlacionadas

CPOD- Bajo Limitación funcional

Índice Gingival - Normal Limitación funcional

Indicadores de Índice Higiene Oral - Muy malo Limitación funcional

Estadístico de correlación $\mathrm{K}$ de Cohen, $\mathrm{p}$ - valor

Estadístico $\mathrm{K}$ de Cohen $-0.019$

$p$ - valor $0.033^{*}$

Estadístico $\mathrm{K}$ de Cohen $-0.08$

$\mathrm{p}$ - valor $0.004^{*}$

Estadístico $\mathrm{K}$ de Cohen $-0.016$

$\mathrm{p}$ - valor ( $\mathrm{HO}-$ Muy Malo $0.022^{*}$

${ }^{*}$ significativo $p<0.05$; NS No Significativo 


\section{DISCUSIÓN}

Según la literatura los movimientos migratorios en diferentes países generan gran expectativa en la valoración del crecimiento de la población en ámbitos de hábitos de salud y la frecuencia de las enfermedades de las personas inmigrantes. ${ }^{14}$

Al investigar Romero $C$ et al en España, la calidad de vida y competencias sociales haciendo un estudio comparativo entre los nativos y los inmigrantes tuvo como resultado que los inmigrantes presentaban menor índice de Calidad de vida además de las dimensiones de bienestar psicológico en relación a los nativos, dichos resultados no son diferentes a los encontrados en nuestra investigación, ya que se relacionan muy directamente porque los indicadores de calidad de vida $\mathrm{y}$ de las dimensiones de Salud oral tuvieron como resultado que la población inmigrante presenta índices desfavorable según los instrumentos de evaluación utilizados. ${ }^{15}$ Asi mismo, Almeida J. en Brasil, el estudio realizado en Chile por Urzua, en Suiza la investigación hecha por Marina K reflejan distintas dificultades proyectadas en poblaciones inmigrantes las que no son ajenas a los resultados de este trabajo de investigación, ya que reflejan similares resultados en componentes de Calidad de vida y Aspectos de salud, los mismo que servirían para mejor dichos índices en las poblaciones afectadas. ${ }^{16,17,18}$

Por otro lado en las investigaciones realizadas en España por Gómez y en Brasil por Da Costa reflejan la importación y el aumento de la frecuencia de enfermedades específicas y que relacionando los resultados de esta investigación presenta similitud porque los índices empleados para el componente de Salud oral refleja el hallazgo de enfermedades como Caries dental y enfermedad periodontal en la población inmigrante estudiada. ${ }^{19,20}$

Por el contrario en Colombia en la investigación realizada por Fernández y que según los resultados reflejan que las condiciones de calidad de vida y salud son favorables para la población inmigrantes no siendo similares a las de esta investigación reflejando que las estrategias sociales utilizadas para con la población estudiada en dicho país son más eficientes. ${ }^{21}$

Finalmente mencionar que según la evidencia científica relacionada con el estudio de la calidad de vida y componentes de salud en poblaciones inmigrantes tienen resultados desfavorables que comprometen el ritmo de vida y el desarrollo físico, social ambiental y psicológico de dicha población, poniendo en riesgo su salud y la de las demás personas que habitan un lugar específico, es por ello la necesidad de establecer estrategias específicas para mejorar dicha condición. ${ }^{22,23,24,25}$

\section{CONCLUSIONES}

La presente investigación muestra un índice CPOD muy bajo, un índice gingival normal y un índice de higiene oral muy malo en la mayor parte la población inmigrante venezolana que formo parte del presente estudio. En relación a la calidad de vida relacionada con la salud oral se obtuvo que la población estudiada tiene buena calidad de vida, representada por más de la mitad de los encuestados. También se aprecia que existe una relación estadísticamente significativa entre la limitación funcional y CPOD bajo, el índice gingival normal y el índice de higiene oral muy malo.

\section{REFERENCIAS BIBLIOGRÁFICAS}

1. Situación en Venezuela [Internet]. UNHCR. [citado 28 de diciembre de 2019]. Disponible en: https://www.acnur. org/situacion-en-venezuela.html

2. Plataforma de Coordinación para Refugiados y Migrantes de Venezuela [Internet]. [citado 28 de diciembre de 2019]. Disponible en: https://r4v.info/es/situations/platform

3. La cifra de migrantes internacionales crece más rápido que la población mundial [Internet]. Noticias ONU. 2019 [citado 28 de diciembre de 2019]. Disponible en: https://news.un.org/es/ story/2019/09/1462242

4. Roura M, Domingo A, Leyva-Moral JM, Pool R. Hispano-Americans in Europe: what do we know about their health status and determinants? A scoping review. BMC Public Health [Internet]. 7 de mayo de 2015 [citado 28 de diciembre de 2019];15. Disponible en: https://www. ncbi.nlm.nih.gov/pmc/articles/PMC4430018/

5. Mouradian WE, Wehr E, Crall JJ. Disparities in children's oral health and access to dental care. JAMA. 22 de noviembre de 2000;284(20):2625-31.

6. Bordoni $N$, Squassi A. LAS MIGRACIONES Y LA SALUD BUCAL EN EL MARCO DE LA SALUD GLOBAL. REFLEXIONES DESDE LA PERSPECTIVA UNIVERSITARIA. Revista de Salud Pública. XX(3):18-31.

7. Silva ES da, Possobon R de F, Barbosa MDM, Batista MJ, Tenuta LMA. Impact of sense of coherence on oral health-related quality of life among Brazilian adults. Braz Oral Res. 2019;33:e100.

8. Koistinen S, Olai L, Ståhlnacke K, Fält A, Ehrenberg A. Oral health-related quality of life and associated factors 
among older people in short-term care. Int J Dent Hyg. 29 de noviembre de 2019;

9. Vasconcelos K, Evangelista S, Silva RA, Oliveira S, Dutra A, Santos A, et al. Assessing the Association between Dental Caries and Nutritional Status in Children from the Brazilian State of Amazonas. Int J Clin Pediatr Dent [Internet]. 2019 [citado 30 de diciembre de 2019];12(4):293-6. Disponible en: https://www.ncbi. nlm.nih.gov/pmc/articles/PMC6898865/

10. Organization WH. Oral Health Surveys: Basic Methods. World Health Organization; 2013. 136 p.

11. Löe H. The Gingival Index, the Plaque Index and the Retention Index Systems. J Periodontol. diciembre de 1967;38(6):Suppl:610-616.

12. Hegde S, Chatterjee E, Rajesh KS, Arun Kumar MS. Obesity and its association with chronic periodontitis: A cross-sectional study. J Educ Health Promot [Internet]. 29 de noviembre de 2019 [citado 30 de diciembre de 2019];8. Disponible en: https://www.ncbi.nlm.nih.gov/pmc/articles/PMC6905353/

13. Greene JC, Vermillion JR. THE SIMPLIFIED ORAL HYGIENE INDEX. J Am Dent Assoc 1939. enero de 1964;68:7-13.

14. González-López JR, Lomas-Campos MM, Rodríguez-Gázquez MA. Evolucion de la inmigracion en Europa y Espana durante los siglos XX y XXI [Internet]. Go.gale.com. 2010 [cited 5 January 2020]. Available from: https://go.gale.com/ps/anonymous?id=GALE\%7CA362169380\& sid=googleScholarE $\mathrm{v}=2.1$ Eit=rElinkaccess $=$ absEissn $=22160973 \mathcal{E} \mathrm{p}=\mathrm{IFMEE}$ sw $=\mathrm{w}$

15. Romero Oliva C, Nunes C, Montilla Coronado C, Lemos I. [Internet]. Scielo.org.co. 2017 [cited 5 January 2020]. Available from: http://www.scielo.org.co/pdf/rups/v16n3/1657-9267rups-16-03-00223.pdf

16. Almeida Weber J, Brunnet A, dos Santos N, Simonetti E, Pizzinato A. [Internet]. Scielo.br. 2019 [cited 5 January 2020]. Available from: http://www.scielo.br/pdf/pusf/v24n1/21753563-pusf-24-01-173.pdf
17. Urzua A, Vega M, Jara A, Trujillo S, Muñoz R. [Internet]. Scielo. conicyt.cl. 2015 [cited 5 January 2020]. Available from: https://scielo.conicyt.cl/pdf/terpsicol/v33n2/art08.pdf

18. Marina K. Salud integral y migración: abordaje transcultural del Proceso Enfermero en un caso clínico del Programa de Salud Migratoria de Ginebra, Suiza [Internet]. Dialnet. 2017 [cited 5 January 2020]. Available from: https://dialnet.unirioja.es/servlet/articulo?codigo $=6195707$

19. Gómez A, Breña L, Sanz S. [Internet]. Scielo.isciii.es. 2019 [cited 5 January 2020]. Available from: http://scielo.isciii.es/pdf/ eg/v18n53/en_1695-6141-eg-18-53-582.pdf

20. Leão LHDC e. [The health of Haitian immigrant workers in Mato Grosso, Brazil: vulnerabilities and risks]. - PubMed - NCBI [Internet]. Ncbi.nlm.nih.gov. 2018 [cited 5 January 2020]. Available from: https://www.ncbi.nlm.nih.gov/pubmed/30726452

21. Fernandez - Niño J, Vasquez A, Flórez V. [Internet]. Scielo.org.co. 2018 [cited 5 January 2020]. Available from: http://www.scielo.org.co/pdf/rsap/v20n4/0124-0064rsap-20-04-530.pdf

22. Almerich-Silla, Jose $\mathcal{E}$ Montiel-Company, Jose. Oral Health Survey of the child population in the Valencia region of Spain. Medicina oral, patología oral y cirugía bucal; 2006. 11. E369-81.

23. Baiju R. et al. Salud oral y calidad de vida: conceptos actuales. India. 2017.

24. Mercédesz O. et al.Calidad de vida relacionada con la salud oral de pacientes sometidos a medicina física y rehabilitación. Turquía. 2018

25. Dominguez C, Crespo C, Vallejo F. [Internet]. Scielo.org.pe. 2019 [cited 5 January 2020]. Available from: http://www.scielo.org.pe/pdf/reh/v29n1/a02v29n1.pdf

\section{CORRESPONDENCIA:}

mvalenzuela@crece.uss.edu.pe 